\title{
$\gamma$-Rays Irradiation Induced Structural and Morphological Changes in Copper Nanowires
}

\author{
H. Shehla, ${ }^{1,2,3,4}$ F. T. Thema, ${ }^{3,4}$ A. Ishaq, ${ }^{1}$ Naveed Zafar Ali, ${ }^{1}$ \\ I. Javed, ${ }^{5}$ D. Wan, ${ }^{6}$ and M. Maaza ${ }^{3,4}$ \\ ${ }^{1}$ National Center for Physics, Quaid-i-Azam University, Islamabad 44000, Pakistan \\ ${ }^{2}$ Center of Excellence in Solid State Physics, University of Punjab, QAC, Lahore 54590, Pakistan \\ ${ }^{3}$ UNESCO-UNISA Africa Chair in Nanoscience/Nanotechnology, College of Graduate Studies, University of South Africa, \\ Muckleneuk Ridge, P.O. Box 392, Pretoria, South Africa \\ ${ }^{4}$ Nanoscience African Network (NANOAFNET), iThemba LABS National Research Foundation, 1 Old Faure Road, \\ P.O. Box 722, Somerset West, Western Cape 7129, South Africa \\ ${ }^{5}$ Department of Physics, Quaid-i-Azam University, Islamabad 44000, Pakistan \\ ${ }^{6}$ School of Materials Science and Engineering, Shanghai University, Shanghai 200444, China
}

Correspondence should be addressed to F. T. Thema; ftthema@gmail.com and A. Ishaq; ishaq@ncp.edu.pk

Received 22 May 2016; Accepted 27 June 2016

Academic Editor: Philippe Caroff

Copyright (c) 2016 H. Shehla et al. This is an open access article distributed under the Creative Commons Attribution License, which permits unrestricted use, distribution, and reproduction in any medium, provided the original work is properly cited.

\begin{abstract}
This contribution reports on the effect of $\gamma$-irradiations on the structural and morphological properties of copper nanowires (CuNWs) within the $\gamma$ doses varying from 6 to $25 \mathrm{kGy}$. At $9 \mathrm{kGy}$, the Cu-NWs started welding, forming perfect X-, V-, II-, and Y-shaped molecular junctions. Further increasing the $\gamma$ dose up to $15 \mathrm{kGy}$ caused the $\mathrm{Cu}-\mathrm{NW}$ s to fuse and form larger diameter NWs. At the highest dose of $25 \mathrm{kGy}$, the nanowires converted into a continuous $\mathrm{Cu}$ thin film. However, X-ray diffraction (XRD) results showed that the structure of the $\mathrm{Cu}-\mathrm{NW}$ s remained stable even after converting into a thin film. The formation of the $\mathrm{Cuprite}\left(\mathrm{Cu}_{2} \mathrm{O}\right)$ phases was observed at higher $\gamma$ dose. The mechanism of forming welded networks of $\mathrm{Cu}-\mathrm{NWs}$ and $\mathrm{Cu}$ thin films is explained via the short and high energy $\gamma$-ray wavelengths which act on $\mathrm{Cu}$ - $\mathrm{Cu}$ molecular covalent bonds isotropically.
\end{abstract}

\section{Introduction}

Metal nanowires made of gold, silver, or copper are promising candidates for future nanodevices as interconnecting circuits which provide electrically conducting paths in view of increasing the device density for future integrated chips [1-6]. Moreover, such metal nanowires are also strong candidates for electrically conductive and transparent electrodes for solar cell applications equivalent to doped zinc oxide [7]. Among other metal nanowires family, the $\mathrm{Cu}-\mathrm{NW}$ s system is a likely contender for the above optoelectronic applications due to its cost-effective and electric performance. While copper $(\rho=1.59 \mathrm{n} \Omega \mathrm{m})$ has an electrical conductivity comparable to that of silver $(\rho=1.67 \mathrm{n} \Omega \mathrm{m})$, it is far cost-effective and more crest-abundant than silver or indium $[8,9]$. In addition, $\mathrm{Cu}$ has a significant presence in the modern semiconductor electronic circuitry. Recently, within the ongoing miniaturization, $\mathrm{Cu}$ is being considered as an electronic interconnector of choice in the nanoscaled devices [10, 11]. Therefore, $\mathrm{Cu}-\mathrm{NWs}$ are attracting considerable interest and are expected to be an essential component of nanoelectronic devices and nanocircuitry in the near future. In space technology, where the environment is generally harsh, such $\mathrm{Cu}$ nanocircuitry has to exhibit an optimal threshold of radiation damage. Consequentially, total radiation dose hardness tolerance testing of future $\mathrm{Cu}-\mathrm{NWs}$ based electronic nanodevices, sensors, circuits, and solar cells working in harsh conditions is an emerging field explored by a broader scientific and engineering communities geared towards space applications. Over long periods of operation, the performance of solar cells and particle detectors in satellites deteriorates when they are exposed to energetic radiations. Considerable effort has been made to study radiation hardness of nanodevices and solar cells [12-15]. Recently, it was shown that $\mathrm{MeV}$ 
carbon ions irradiations on silver NWs (Ag-NWs) induce significant damage on their structure while on the contrary they are stable under $\mathrm{MeV}$ proton beams [16, 17]. CuNWs are potential future integrated electronic components in nanodevices which require hardness tolerance testing. Therefore, our aim is to systematically study the effects of radiation on the properties of $\mathrm{Cu}-\mathrm{NW}$. There is currently limited information in the broader literature dealing with the radiation effects on $\mathrm{Cu}-\mathrm{NW}$ s especially $\gamma$-radiation $[18,19]$. As for the protons from deep space radiations, $\gamma$-photons are also abundant in upper space which may cause ionization and radiation damage on embarked $\mathrm{Cu}-\mathrm{NW}$ s based nanodevices. The study of $\gamma$-radiations effects on $\mathrm{Cu}-\mathrm{NWs}$ is therefore necessary. In this paper, $\gamma$-radiations effect on the structure and morphology of Cu-NWs is discussed systematically.

\section{Experimental}

The Cu-NWs used in this work were purchased from the Guangzhou Jiechuang Trading Co. Ltd., China. CuNWs were dispersed in isopropanol with concentration of $20 \mathrm{mg} / \mathrm{mL}$ and then spray-coated on glass substrates placed on a hot plate at $170^{\circ} \mathrm{C}$ for rapid evaporation of isopropanol. $\mathrm{Cu}-\mathrm{NWs}$ networks were formed on glass substrate and cut into $1 \times 1 \mathrm{~cm}^{2}$ dimensions to ensure that all samples have the same physical properties. For TEM study, few drops of $\mathrm{Cu}-$ NWs solution were dispersed on a copper grid. All samples were then exposed to $5 \mathrm{MeV} \gamma$-photons at various doses of 6 , $9,12,15$, and $25 \mathrm{kGy}$ using a $\mathrm{Co}^{60}$ source at room temperature and under atmospheric pressure. The dose rate of the $\gamma$ source was $38 \mathrm{~Gy} / \mathrm{min}$. The time of exposure for different doses was calculated from the activity of the source, as given in Table 1.

The samples were characterized using multiple techniques such as high resolution scanning electron microscopy (HRSEM), EDX, high resolution transmission electron microscopy (HRTEM), and X-ray diffraction (XRD) techniques. The structural study of unirradiated and $\gamma$-irradiated samples with varying irradiation doses, that is, $6-25 \mathrm{kGy}$, was investigated by both qualitative and quantitative Rietveld refinement of the high resolution $\mathrm{X}$-ray powder diffraction (XRD) data. The XRD data was collected in the $2 \theta$ angular range from $29^{\circ}$ to $80^{\circ}$ with an integration time of $2 \mathrm{~s}$ per 0.02 steps, employing a Bruker X-ray diffractometer with $\mathrm{Cu}_{\mathrm{K} \alpha}$ radiation $(1.5409 \AA)$. TOPAS program (version 4.1 ) is utilized for structural refinements using Rietveld refinement technique [19].

\section{Results and Discussion}

Structural study of unirradiated and $\gamma$-irradiated Cu-NWs was characterized by XRD technique. Figure 1 shows the XRD pattern of samples exposed at different doses. Figure 1(a) shows the XRD pattern of unirradiated $\mathrm{Cu}-\mathrm{NW}$. The XRD pattern comprising one (111) peak at $2 \theta=44.2^{\circ}$ which is preferred crystal plan of $\mathrm{Cu}-\mathrm{NW}$. Other two low intensities peaks at $2 \theta=52.4^{\circ}$ and $73.9^{\circ}$ are corresponding to the crystal planes (200) and (220), respectively. These peaks and intensities show that $\mathrm{Cu}-\mathrm{NW}$ s had a polycrystalline structure.
TABLE 1: Exposure time for different doses.

\begin{tabular}{lc}
\hline Dose $(\mathrm{kGy})$ & Time of exposure \\
\hline 6 & $2 \mathrm{~h} 37 \mathrm{~min} 8 \mathrm{~s}$ \\
9 & $3 \mathrm{~h} 57 \mathrm{~min} 0 \mathrm{~s}$ \\
12 & $5 \mathrm{~h} 15 \min 6 \mathrm{~s}$ \\
15 & $6 \mathrm{~h} 34 \min 8 \mathrm{~s}$ \\
18 & $7 \mathrm{~h} 53 \min 7 \mathrm{~s}$ \\
21 & $9 \mathrm{~h} 12 \min 6 \mathrm{~s}$ \\
25 & $10 \mathrm{~h} 58 \min 2 \mathrm{~s}$ \\
\hline
\end{tabular}

XRD results are confirmed with the HRTEM images shown in Figure 2(a).

$\mathrm{X}$-ray powder diffraction patterns of unirradiated and $\gamma$ irradiated Cu-NWs samples were fitted using Rietveld refinements and the difference curves. There was no significant variation in lattice constant " $a$ " and unit cell volume " $V$ " for both unirradiated and $\gamma$-irradiated samples. The lattice parameter " $a$ " of the Cu-NWs was found to be $3.610 \AA$ for all the four samples as shown in Figure 2(b). The Rietveld refinement of unirradiated and irradiated $\mathrm{Cu}-\mathrm{NWs}$ at a dose of $9 \mathrm{kGy}$ shows that for the entire powder diffraction pattern no impurities were observed (Figure 1(b)). However, at higher irradiation doses (12 kGy and $15 \mathrm{kGy}$ ), surface microstructure evolutions start taking place and it turns out that, apart from standard $\mathrm{Cu}-\mathrm{NW}$ s diffraction peaks, additional reflections started appearing, which can be indexed in terms of a mixture of metallic copper and cubic $\mathrm{Cu}_{2} \mathrm{O}$ [20].

The weight percentages of both the phases are retrieved from program Topas 4.1 using quantitative multicomponent Rietveld analyses [21]. The Rietveld refinement results showed that a small fraction (15\%) of impurity Cuprite phase $\left(\mathrm{Cu}_{2} \mathrm{O}\right.$ ) (space group $P n-3 m S, a=0.4265 \mathrm{~nm}$ ) is also present in the XRD patterns of samples in Figures 1(c) and 1(d), apart from major (85\%) face centered cubic (fcc) Cu (space group Fm-3m, $a=0.3615 \mathrm{~nm}$ ) phase. Moreover, as the dose increases, there is a slight increase in the intensity of (111) peak of fcc $\mathrm{Cu}$ lattice along with decrease in full width half maximum (FWHM) beckoning the increase in crystallinity of the sample. Recently, irradiation effects were studied on InGaN epilayer thin film and observed improvement of crystal quality [22].

In addition, the XRD patterns of unirradiated and $\gamma$ irradiated $\mathrm{Cu}-\mathrm{NW}$ s samples were refined in the space group $F m-3 m$ with $\mathrm{Cu}$ at $4 \mathrm{a} 0,0,0$, whereas the impurity phase $\mathrm{Cu}_{2} \mathrm{O}$ was refined in space group $P n-3 m S$ with $\mathrm{Cu}$ residing at $4 \mathrm{~b} 1 / 4,1 / 4,1 / 4$ site and oxygen occupying the $2 \mathrm{a} 0,0$, 0 site. During the refinement, all atomic positions were fully occupied, and isotropic thermal vibration parameters and occupancies were found to be highly correlated. The weighted profile $R$ factor $\left(R_{\mathrm{wp}}\right.$ and $\left.R_{\text {exp }}\right)$ values show a good agreement between the experimental model and respective X-ray diffraction data. The $R_{\mathrm{wp}}$ values of Figures 1(a) to 1(d) are $5.84,10.60,3.61$, and 2.79 , respectively, while the $R_{\text {exp }}$ values of Figures 1 (a) to $1(\mathrm{~d})$ show similar behavior and are $3.76,4.44,2.80$, and 132.44 , respectively. 


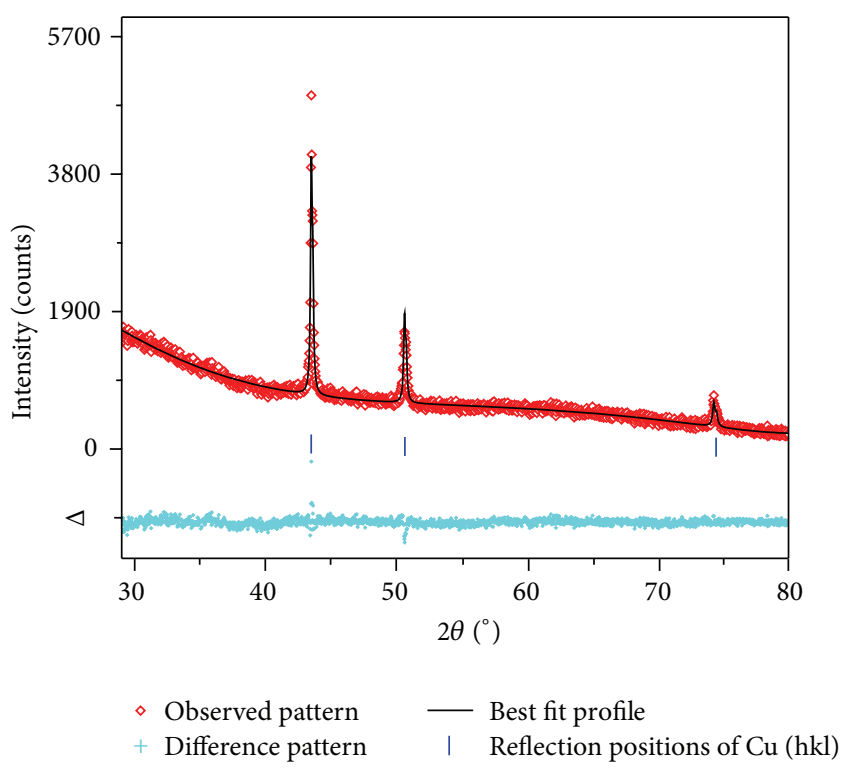

(a)

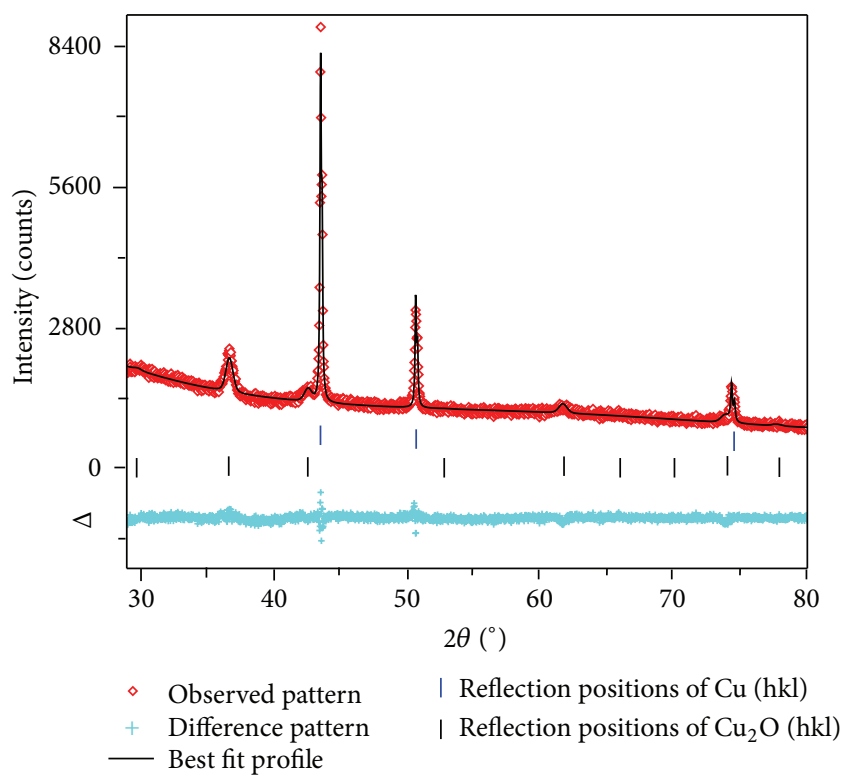

(c)

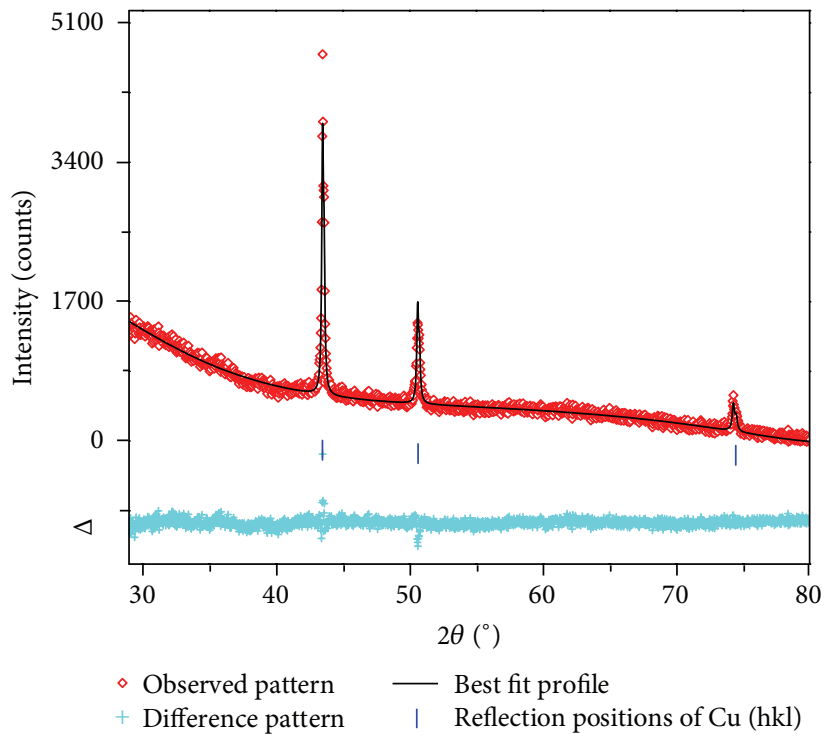

(b)

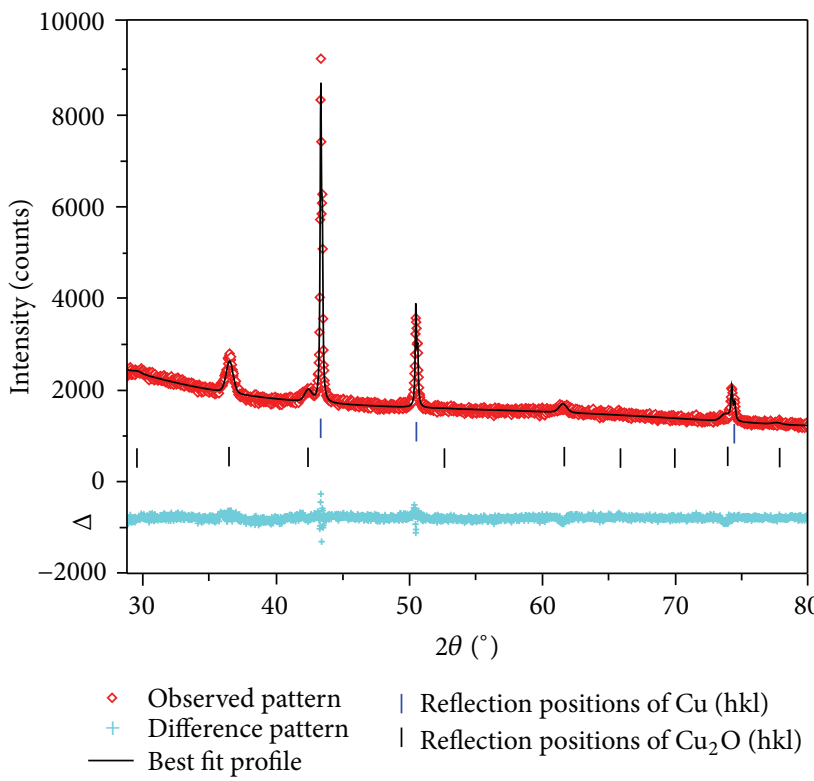

(d)

FIGURE 1: XRD spectra of Cu-NWs (a) unirradiated, (b) irradiated at $9 \mathrm{kGy}$, (c) irradiated at $12 \mathrm{kGy}$, and (d) irradiated at $15 \mathrm{kGy}$.

The morphological changes of the $\mathrm{Cu}-\mathrm{NW}$ s were studied after $\gamma$-irradiation. Figure 3(a) shows typical HRSEM images of unirradiated $\mathrm{Cu}-\mathrm{NWs}$. The diameters of unirradiated $\mathrm{Cu}-$ NWs range from 100 to $150 \mathrm{~nm}$. Moreover, it can be observed from Figure 3(a) that the $\mathrm{Cu}-\mathrm{NWs}$ are covered with an amorphous $\mathrm{Cu}$ structure on the outer sides. Figure $3(\mathrm{~b})$ is the corresponding EDX of unirradiated $\mathrm{Cu}-\mathrm{NWs}$. The $\mathrm{Cu}-$ NWs were first irradiated with $\gamma$-irradiation to a dose of $6 \mathrm{kGy}$. It can be observed that coalescence of these NWs, called necking, commenced as shown in Figure 3(c). Further increase in $\gamma$ dose up to $9 \mathrm{kGy}$ resulted in nanowelding between two $\mathrm{Cu}-\mathrm{NW}$ s and $\mathrm{X}$-, Y-, II-, and V-shaped junctions between the $\mathrm{Cu}-\mathrm{NWs}$ were formed by $\gamma$-irradiation induced coalescence (Figure 4) because as their molecular bonding is broken, the spacing between atoms decreases; hence, their surface area increases. Schematic mechanism of the coalescence process is presented in Figure 5 where the process is started from necking to joining of $\mathrm{Cu}-\mathrm{NWs}$ when subjected to medium $\gamma$-fluence and finally to complete coalescence of two NWs at high $\gamma$-fluence, forming single NWs with double diameter. Recently, coalescence of silver nanowires by carbon ion beam irradiation has been reported and formed X-, Y-, and II-shaped molecular junctions [16]. Formation of X-, Y-, $\mathrm{V}$-, and II-shaped junctions is also confirmed from HRTEM images in Figure 6 (indicated by arrows). Welding of Cu-NWs became visible due to the strong and high energy short $\gamma$-rays induced, showing clearly the formation of junctions, which ultimately resulted in welded $\mathrm{Cu}-\mathrm{NW}$ networks. However, 


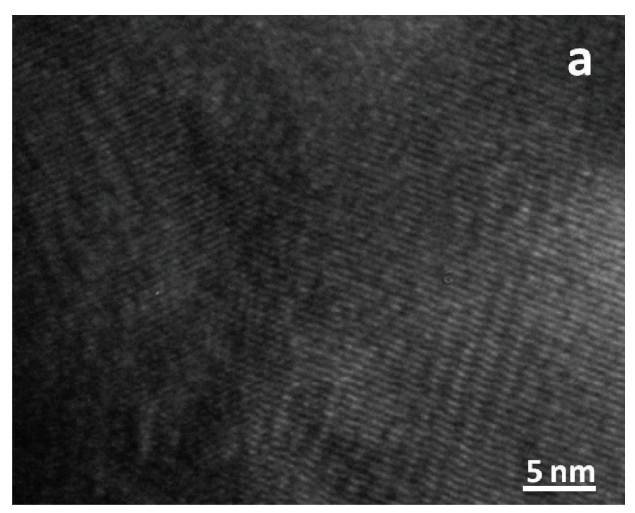

(a)

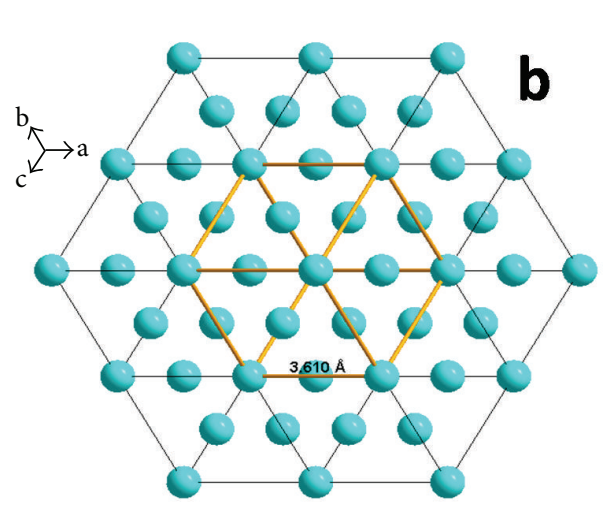

(b)

Figure 2: (a) HRTEM image of Cu-NWs. (b) Crystal structure with unit cell (orange sticks) of $3 \times 3 \times 3$ supercell of face centered cubic lattice of copper nanowire (space group $F m-3 m, a=0.3615 \mathrm{~nm}$ ) viewed along the [111] direction.

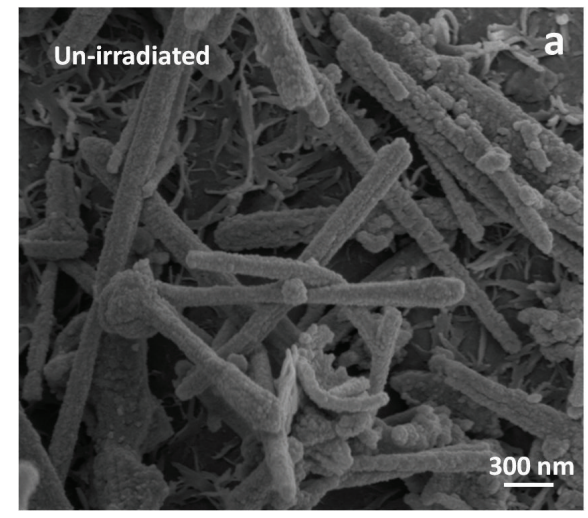

(a)

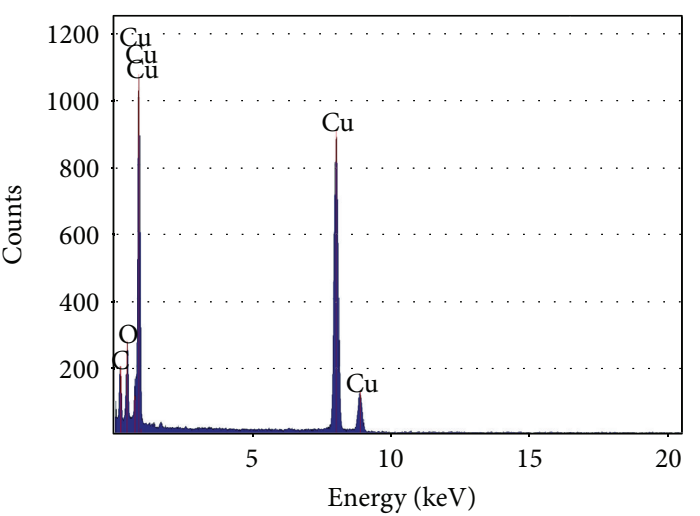

- Acquiring EDX

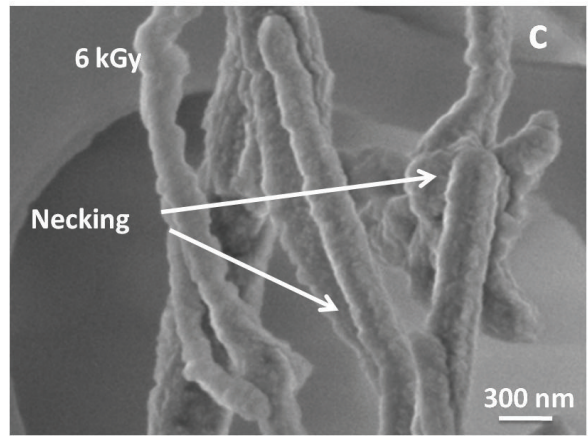

(c)

FIGURE 3: SEM images of Cu-NWs (a) unirradiated and (b) corresponding EDX spectra (c) irradiated at $6 \mathrm{kGy}$ ions $/ \mathrm{cm}^{2}$.

the morphology and structure of the nanowires remained unchanged. It is clear from Figures 4 and 6 that, after $\gamma$ irradiation, different types of junctions are formed between $\mathrm{Cu}-\mathrm{NW}$ s whenever these NWs overlap.

Further increase in $\gamma$-irradiation dose up to $12 \mathrm{kGy}$ resulted in coalescence at junction positions and perfect nanowelding of $\mathrm{Cu}-\mathrm{NW}$ s is obtained and shown in Figure 7. HRTEM images are consistent with XRD results (Figure 8). Moreover, perfect nanowelding of crystalline structure was achieved by $\gamma$-rays induced, as shown in Figure 8. $\gamma$ irradiation induced nanowelding of $\mathrm{Cu}-\mathrm{NWs}$ is a new approach to integrate any nanodevices or forming welded 

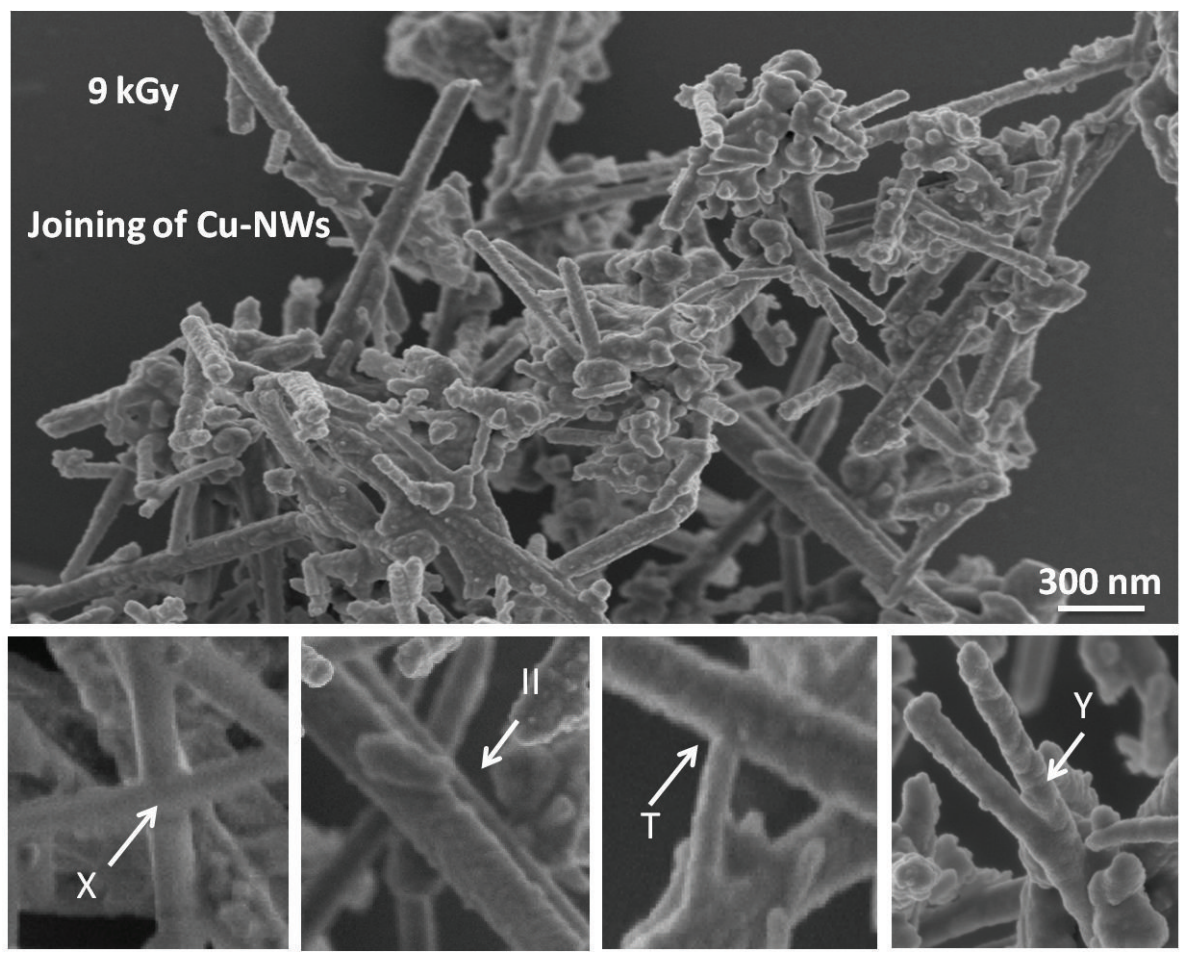

Figure 4: SEM image of Cu-NWs irradiated at a dose of $9 \mathrm{kGy}$ : X-, Y-, V-, and II-shaped junctions are observed.

Coalescence process

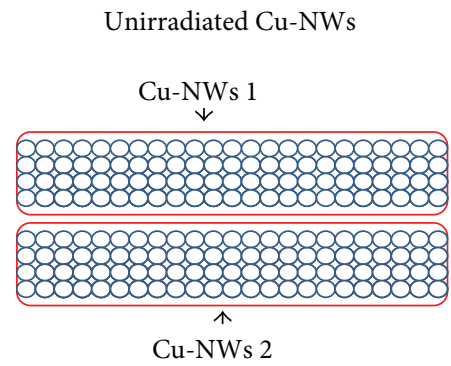

Medium dose gamma irradiation

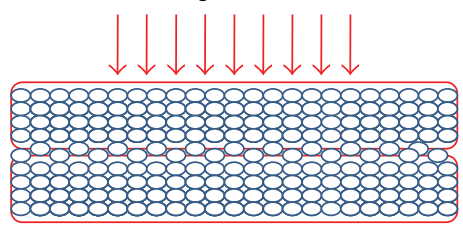

Joining

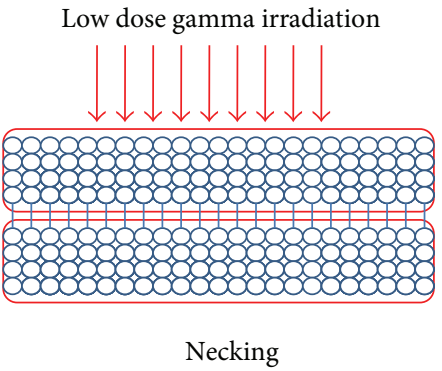

High dose gamma irradiation

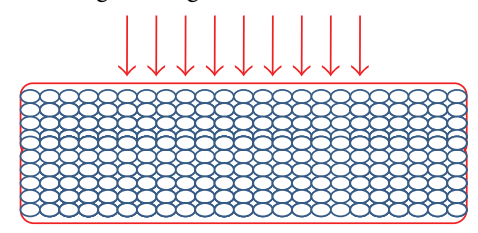

Coalescence

FIGURE 5: Gamma irradiation induced coalescence process.

networks for solar cells. The welding of Ag-NWs has matured for fabrication of transparent electrodes for solar cells [16] while work on welded networks of Cu-NWs as a transparent electrode still needs to be explored. Our new approach to get welded conducting networks of $\mathrm{Cu}-\mathrm{NWs}$ will be useful for solar cell designers and integration of nanodevices.

To study the radiation hardness of $\mathrm{Cu}-\mathrm{NWs}$ under $\gamma$ rays with high dose, results are mentioned in Figures 9 and 10. At an irradiation dose of $15 \mathrm{kGy}$, the $\mathrm{Cu}-\mathrm{NWs}$ start to change their morphology and convert into a thin $\mathrm{Cu}$ film (Figure 9(a)). Figure 9(b) corresponds to HRTEM image which shows $\mathrm{Cu}-\mathrm{NWs}$ coming closer to each other and converting into bulk material. Figure 10(a) clearly shows that molecular bond breakage of $\mathrm{Cu}-\mathrm{NWs}$ results in intact bulk material. The corresponding TEM image is consistent with SEM as shown in Figure 10(b). Further increase of dose to $25 \mathrm{kGy}$ converts the whole nanostructure to $\mathrm{Cu}$ thin film as shown in Figure 10(c). It can be concluded that $\mathrm{Cu}-\mathrm{NWs}$ 


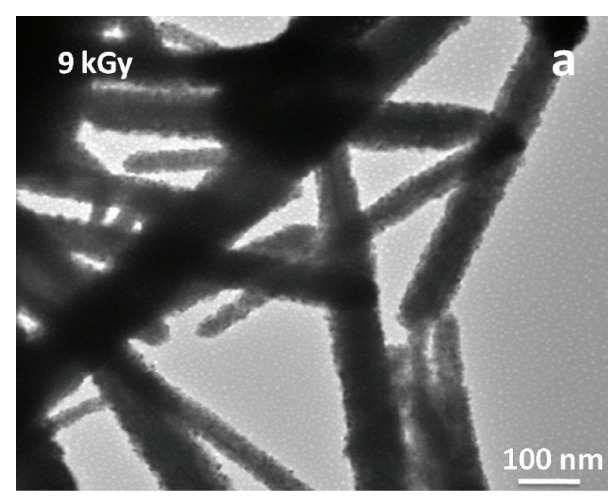

(a)

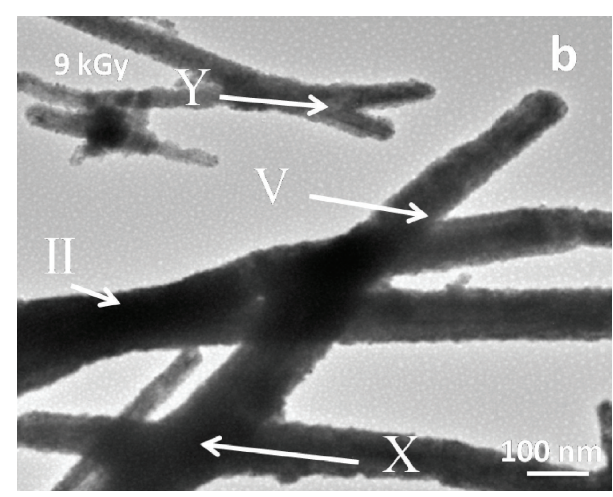

(b)

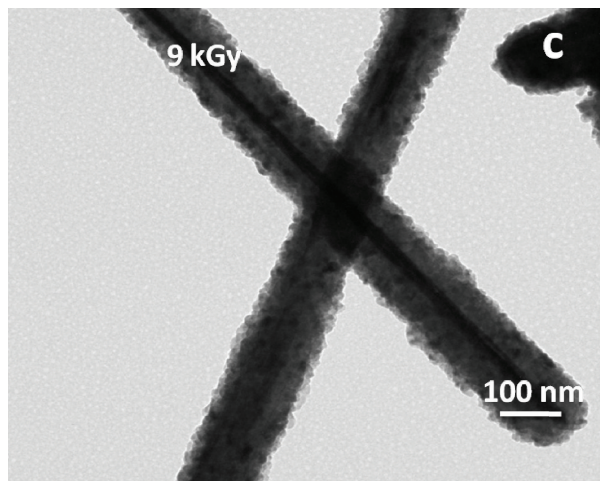

(c)

Figure 6: ((a)-(c)) TEM images of Cu-NWs irradiated at a dose of 9 kGy: X-, Y-, V-, and II-shaped junctions are observed.

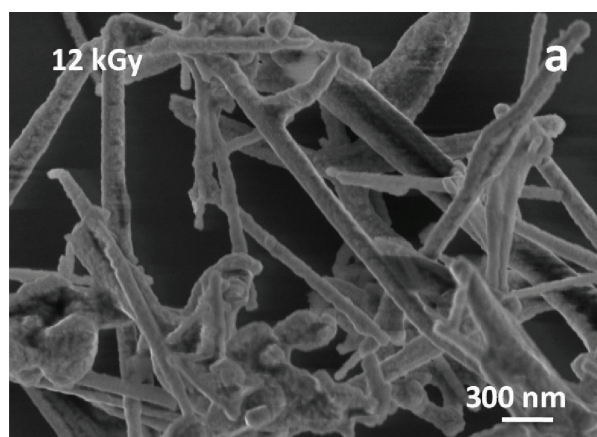

(a)

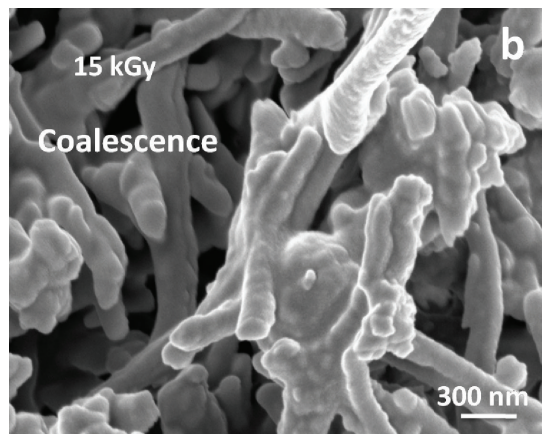

(b)

Figure 7: SEM image of Cu-NWs (a) irradiated at $12 \mathrm{kGy}$ and (b) irradiated at $15 \mathrm{kGy}$.

based nanodevices or solar cells may not be suitable materials for use in harsh environment.

A detailed discussion of the observed nanowelding and conversion of $\mathrm{Cu}-\mathrm{NW}$ s into $\mathrm{Cu}$ thin film appears hereunder. At a dose of $9 \mathrm{kGy}$, welding of $\mathrm{Cu}-\mathrm{NW}$ s became visible due to the breakage $\mathrm{Cu}-\mathrm{Cu}$ bonds by the $\gamma$-rays which are isotropically distributed, showing clearly the formation of junctions, which ultimately resulted in welded $\mathrm{Cu}-\mathrm{NW}$ networks. However, the morphology and structure of the nanowires remained unchanged. Nanowelding process is discussed in schematic diagram as shown in Figure 5. The figure shows that, at low $\gamma$-fluence, necking between nanowires is started.
At medium $\gamma$-fluence, coalescence starts and at higher fluence $\mathrm{Cu}-\mathrm{NWs}$ fused on each other. Finally, Cu-NWs form four types of molecular junctions (i.e., X-, Y-, T-, and II-shaped junctions). The breakage of $\mathrm{Cu}-\mathrm{NW}$ s was conducted using the $\gamma$-irradiation which acted only on the covalent bonds leaving the structure intact as evidenced by the XRD. $\gamma$-Irradiation in $\mathrm{MeV}$ energy range interacted with target material and suffers significant electronic energy loss along its paths in the target material and this attracted addition of oxygen functional groups from the atmosphere resulting in the formation of $\mathrm{Cu}_{2} \mathrm{O}$ material as evidenced by XRD (Figures $1(\mathrm{c})$ and $1(\mathrm{~d})$ ). Moreover, this $\gamma$-irradiation induced phase changes which 


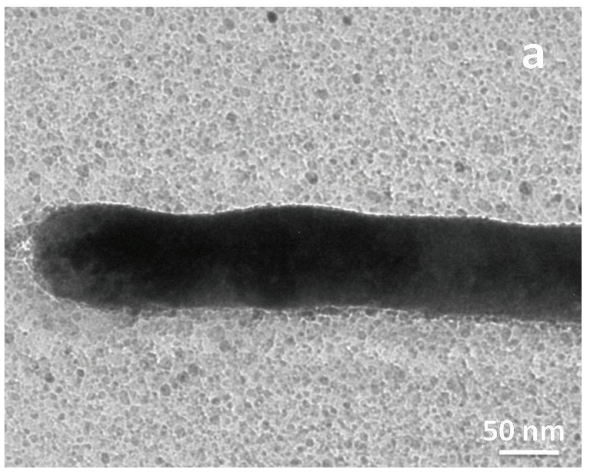

(a)

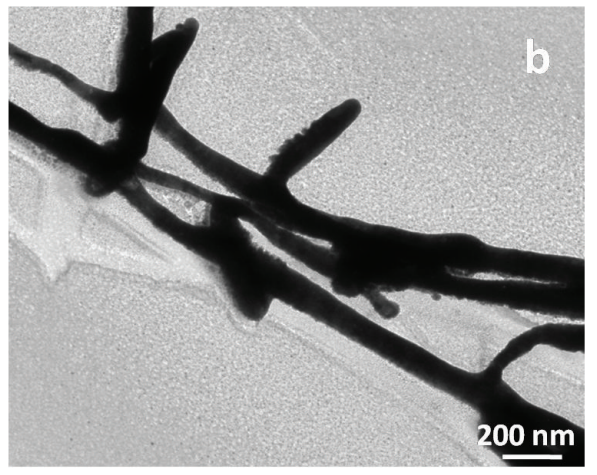

(b)

Figure 8: ((a) and (b)) SEM images of Cu-NWs irradiated at a dose of $15 \mathrm{kGy}$.

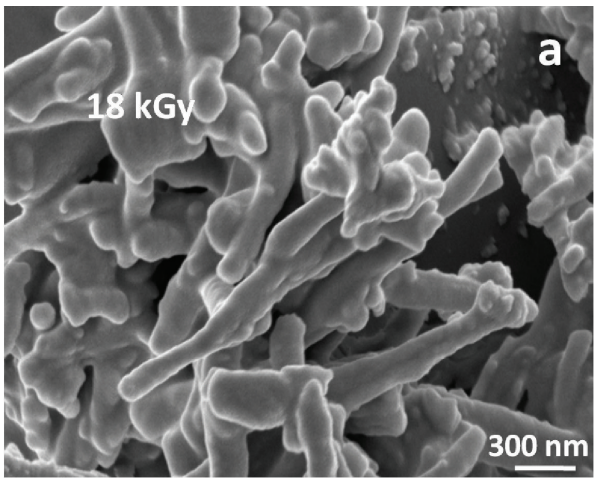

(a)

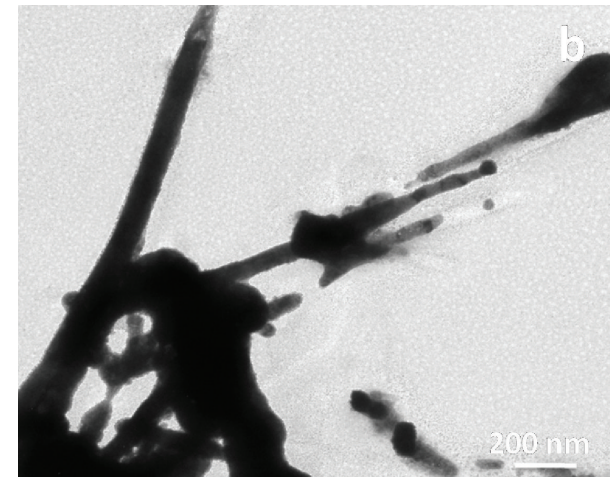

(b)

FIGURE 9: (a) SEM image of Cu-NWs irradiated at a dose of $18 \mathrm{kGy}$ (b) Corresponding TEM image.

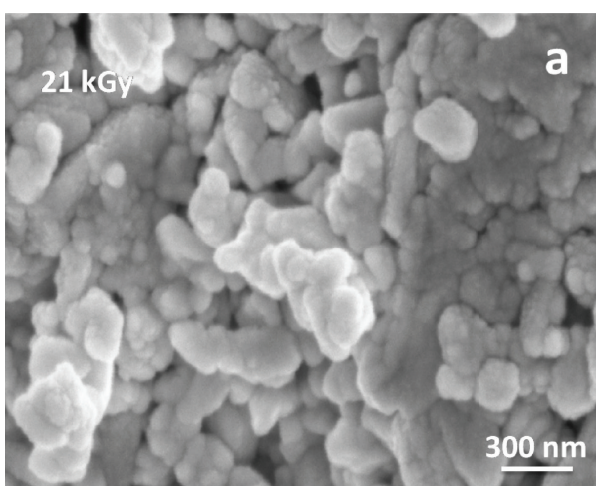

(a)

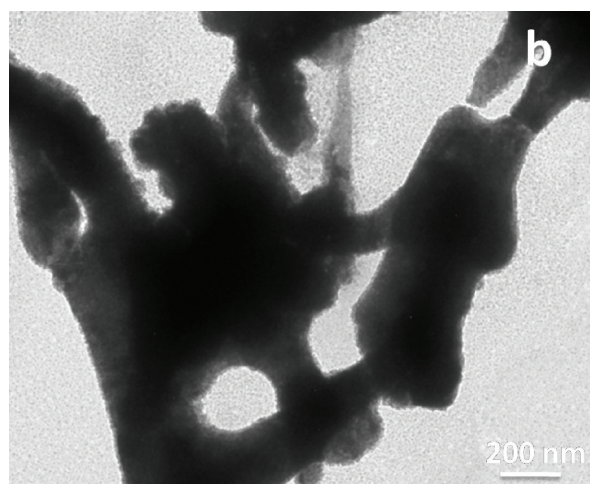

(b)

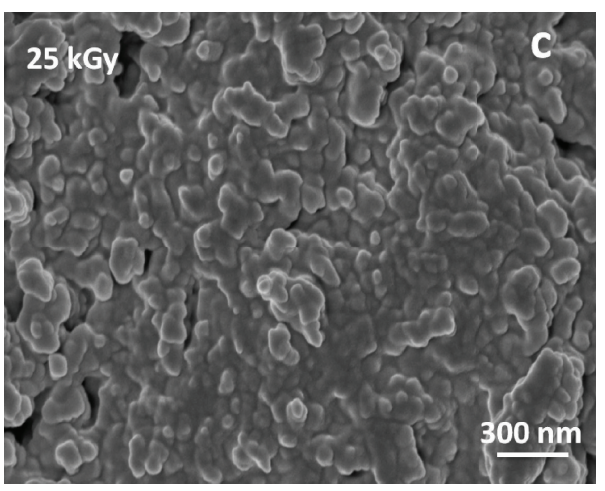

(c)

FIGURE 10: SEM image of Cu-NWs (a) irradiated at $21 \mathrm{kGy}$. (b) Corresponding TEM image (c) irradiated at $25 \mathrm{kGy}$. 
at room temperature do not disorientate the coordination of the $\mathrm{Cu}$ atoms due to breakage of bonds resulting in atomic closeness and mass movement at contact positions of $\mathrm{Cu}$ NWs to reach their equilibrium stability and changing of position but maintaining the initial orientation of the $\mathrm{Cu}$ atomic structure resulting in a sheet-like surface area. Atoms are never displaced by $\gamma$-irradiation induced effect since $\gamma$ rays only act on bonds. As a preconclusion, this study will be followed by the investigation of the effect of other radiations on similar samples using a similar approach as in other systems that we studied [23].

\section{Conclusion}

In conclusion, we have demonstrated a nanowelding technique in which large scale molecular junctions of $\mathrm{Cu}$ NWs were formed by $\gamma$-irradiation. These nanowires were welded on each other due to isotropic $\gamma$-ray irradiated metal $\mathrm{Cu}-\mathrm{NWs}$. This technique is very useful for fabrication of large scale junction-like structures comprising welded $\mathrm{Cu}$ nanowires with FCC cubic phase with uniform diameters. It is believed that the synthesis of connections between metallic nanowires is very important in nanoelectronics and circuit industry. It can be concluded that $\mathrm{Cu}$ nanowires may not be suitable materials for use in harsh environment like nanodevices or solar cells for upper space applications because $\mathrm{Cu}-\mathrm{NW}$ in their pure form cannot maintain their nanoidentity under harsh environments.

\section{Disclosure}

Plagiarism test has been carried out via ID 555843245 (similarity index 09\%) in Turnitin software.

\section{Competing Interests}

The authors declare that they have no competing interests.

\section{Acknowledgments}

iThemba LABS, UNESCO-UNISA, TWAS, NCP, and higher education commission of Pakistan are gratefully acknowledged.

\section{References}

[1] Y. Li, F. Qian, J. Xiang, and C. M. Lieber, "Nanowire electronic and optoelectronic devices," Materials Today, vol. 9, no. 10, pp. $18-27,2006$.

[2] Y. Cui, Q. Wei, H. Park, and C. M. Lieber, "Nanowire nanosensors for highly sensitive and selective detection of biological and chemical species," Science, vol. 293, no. 5533, pp. 1289-1292, 2001.

[3] J. I. Pascual, J. Mendez, J. Gomezherrero et al., "Properties of metallic nanowires from conductance quantization to localization," Sci, vol. 267, no. 5205, pp. 1793-1795, 1995.

[4] B. H. Hong, S. C. Bae, C.-W. Lee, S. Jeong, and K. S. Kim, "Ultrathin single-crystalline silver nanowire arrays formed in an ambient solution phase," Science, vol. 294, no. 5541, pp. 348 $351,2001$.

[5] Y. Kondo and K. Takayanagi, "Synthesis and characterization of helical multi-shell gold nanowires," Science, vol. 289, no. 5479, pp. 606-608, 2000.

[6] J. C. González, V. Rodrigues, J. Bettini et al., "Indication of unusual pentagonal structures in atomic-size $\mathrm{Cu}$ namwires," Physical Review Letters, vol. 93, no. 12, Article ID 126103, 2004.

[7] J. Kennedy, A. Markwitz, H. J. Trodahl, B. J. Ruck, S. M. Durbin, and W. Gao, "Ion beam analysis of amorphous and nanocrystalline group III-V nitride and $\mathrm{ZnO}$ thin films," Journal of Electronic Materials, vol. 36, no. 4, pp. 472-482, 2007.

[8] S. Yun, X. Niu, Z. Yu, W. Hu, P. Brochu, and Q. Pei, "Compliant silver nanowire-polymer composite electrodes for bistable large strain actuation," Advanced Materials, vol. 24, no. 10, pp. 13211327, 2012.

[9] H. Koga, M. Nogi, N. Komoda, T. T. Nge, T. Sugahara, and K. Suganuma, "Uniformly connected conductive networks on cellulose nanofiber paper for transparent paper electronics," NPG Asia Materials, vol. 6, article e93, 2014.

[10] Y. Shi, H. Li, L. Chen, and X. Huang, "Obtaining ultra-long copper nanowires via a hydrothermal process," Science and Technology of Advanced Materials, vol. 6, no. 7, pp. 761-765, 2005.

[11] B. Wang, Y. Xu, K.-L. Yung, W. Chen, and C.-L. Kang, "Aligning and soldering pure-copper nanowires for nanodevice fabrication," Journal of Microelectromechanical Systems, vol. 22, no. 3, pp. 519-526, 2013.

[12] Y. Eisen, L. G. Evans, S. Floyd, C. Schlemm, R. Starr, and J. Trombka, "Radiation damage of Schottky CdTe detectors irradiated by $200 \mathrm{MeV}$ protons," Nuclear Instruments and Methods in Physics Research, vol. 491, no. 1-2, pp. 176-180, 2002.

[13] M. Zanarini, P. Chirco, W. Dusi et al., "Radiation damage induced by $2 \mathrm{MeV}$ protons in CdTe and CdZnTe semiconductor detectors," Nuclear Instruments and Methods in Physics Research Section B: Beam Interactions with Materials and Atoms, vol. 213, pp. 315-320, 2004.

[14] N. H. Lee, J. W. Cho, S. H. Kim, J. H. Ha, and Y. H. Kim, "A study on a radiation hardening of an infrared detector," Journal of Nuclear Science and Technology, vol. 45, pp. 564-567, 2008.

[15] D. L. Batzner, A. Romeo, M. Dobeli, H. Zogg, and A. N. Tiwari, "Radiation hardness of CdTe/CdS solar cells," in Proceedings of the e 17th EC PVSEC Munich, Munich, Germany, October 2001.

[16] B. Bari, S. Honey, M. Morgan et al., "MeV carbon ion irradiation-induced changes in the electrical conductivity of silver nanowire networks," Current Applied Physics, vol. 15, no. 5, article no. 3894, pp. 642-647, 2015.

[17] S. Honey, S. Khan, J. Iqbal et al., "Protons irradiation induced coalescence of silver nanowires," Current Nanoscience, vol. 11, no. 6, pp. 792-796, 2015.

[18] V. J. Kennedy, A. Markwitz, U. D. Lanke, A. McIvor, H. J. Trodahl, and A. Bittar, "Ion beam analysis of ion-assisted deposited amorphous GaN," Nuclear Instruments and Methods in Physics Research Section B: Beam Interactions with Materials and Atoms, vol. 190, no. 1-4, pp. 620-624, 2002.

[19] A. A. Coelho, "Indexing of powder diffraction patterns by iterative use of singular value decomposition," Journal of Applied Crystallography, vol. 36, no. 1, pp. 86-95, 2003.

[20] M. Madhuku, G. Husnain, I. Ahmad, and H. Saleem, "Irradiation-induced improvement in crystalline quality of epitaxially grown InGaN thin films," in Proceedings of the A Preliminary Study, Proceedings of SAIP, pp. 59-65, 2014. 
[21] A. Kirfel and K. D. Eichhorn, "Accurate structure analysis with synchrotron radiation. The electron density in $\mathrm{Al}_{2} \mathrm{O}_{3}$ and $\mathrm{Cu}_{2} \mathrm{O}$," Acta Crystallographica Section A, vol. 46, no. 4, pp. 271284, 1990.

[22] A. Markwitz, V. J. Kennedy, and H. Baumann, "Formation of micrometer sized crater shaped pits in silicon by low-energy ${ }^{22} \mathrm{Ne}^{+}$implantation and electron beam annealing," Nuclear Instruments and Methods in Physics Research, Section B, vol. 206, pp. 179-183, 2003.

[23] F. T. Thema, P. Beukes, Z. Y. Nuru et al., "Physical properties of graphene via $\gamma$-radiolysis of exfoliated graphene oxide," in Proceedings of the 7th International Symposium on Macro- and Supramolecular Architectures and Materials, vol. 2, no 7, pp. 4038-4045, 2015. 

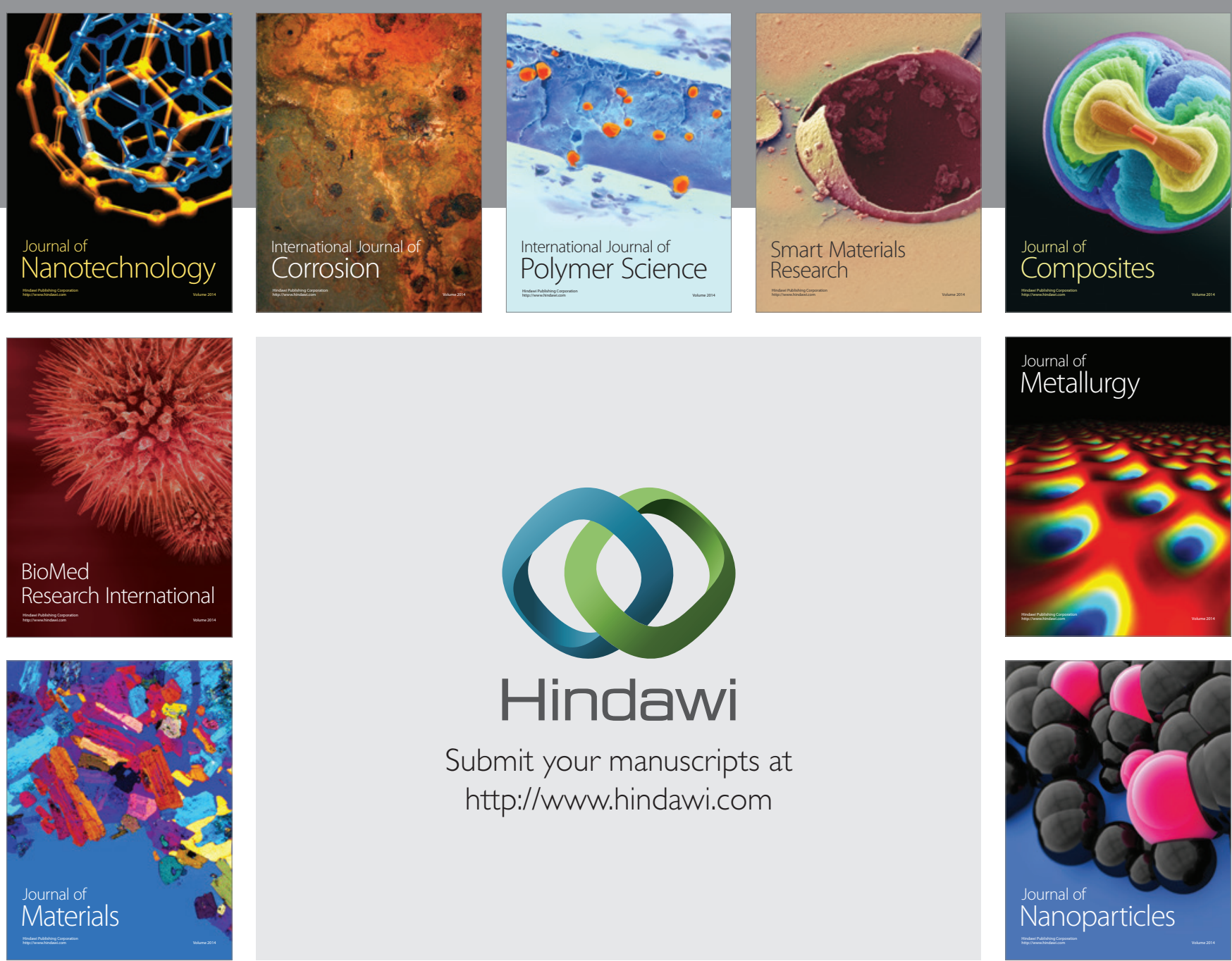

\section{Hindawi}

Submit your manuscripts at

http://www.hindawi.com

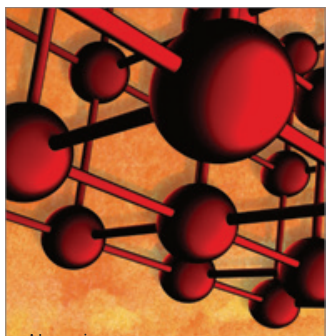

Materials Science and Engineering
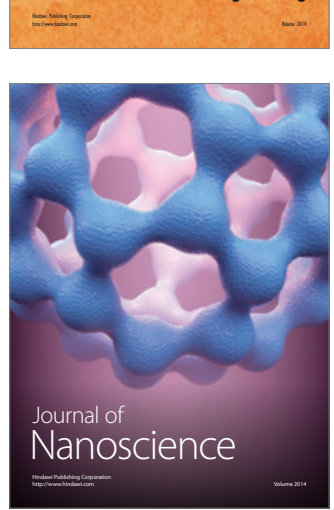
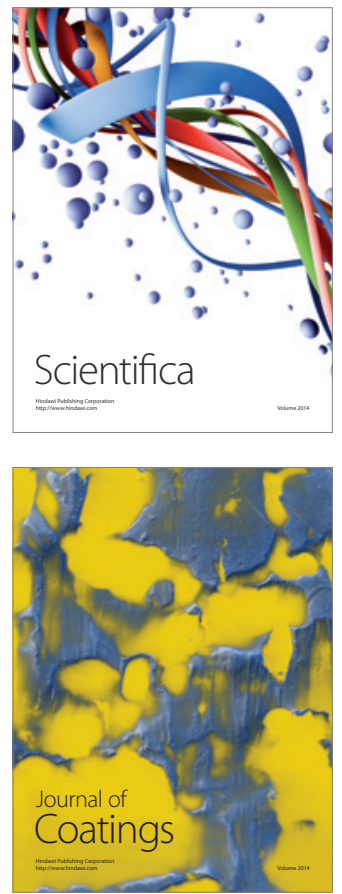
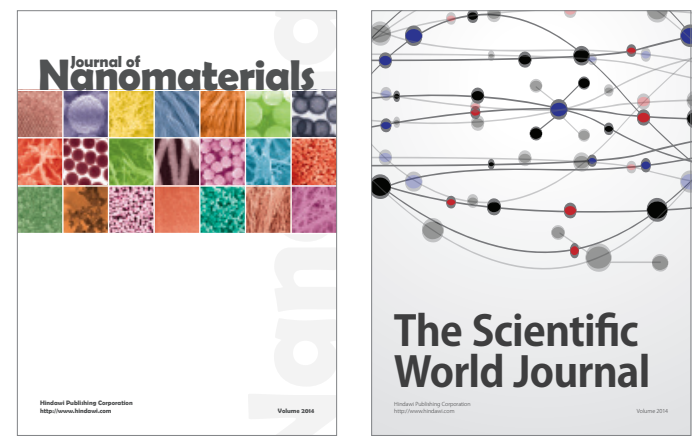

The Scientific World Journal
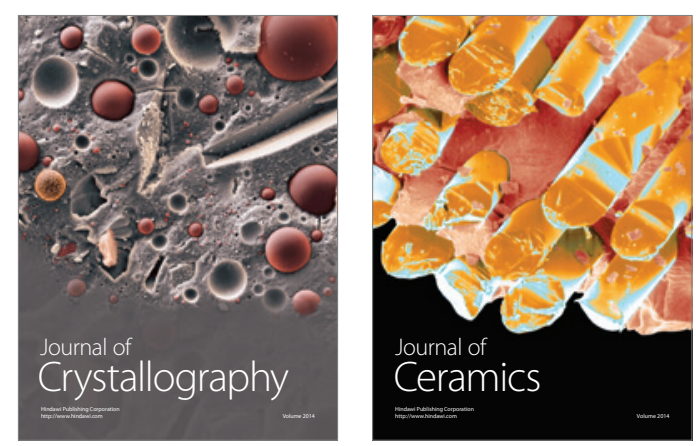
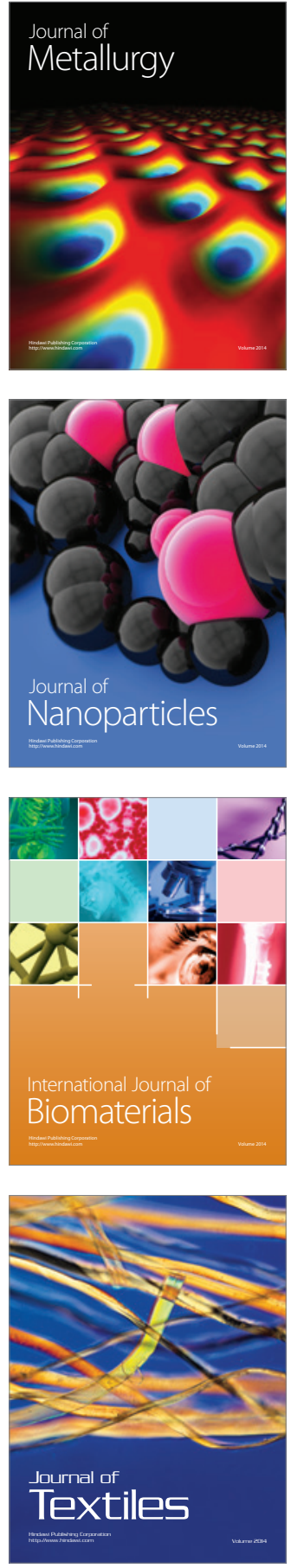